\title{
Facile preparation of silver nanoparticles in bulk silicate glass by high-repetition-rate picosecond laser pulses
}

\author{
Danyang Shen (沈丹阳) $)^{1,2}$, Jing Qian (钱 静) ${ }^{1,2}$, Chengwei Wang (王承伟) ${ }^{1,2}$, Guande Wang (王关德) $)^{1,2}$ ， \\ Xuehui Wang (王雪辉) ${ }^{3}$, and Quanzhong Zhao (赵全忠) ${ }^{1,2^{*}}$ \\ ${ }^{1}$ State Key Laboratory of High Field Laser Physics, Shanghai Institute of Optics and Fine Mechanics, Chinese Academy of Sciences, Shanghai 201800 , China \\ ${ }^{2}$ Center of Materials Science and Optoelectronics Engineering, University of Chinese Academy of Sciences, Beijing 100049, China \\ 3 Technology Center, Huagong Laser Engineering Co., Ltd., Wuhan 430000, China
}

*Corresponding author: zqz@siom.ac.cn

Received June 28, 2020 | Accepted September 4, 2020 | Posted Online December 2, 2020

\begin{abstract}
One-step precipitation of $\mathrm{Ag}$ nanoparticles in $\mathrm{Ag}^{+}$-doped silicate glasses was achieved through a focused picosecond laser with a high repetition rate. Absorption spectra and transmission electron microscopy (TEM) confirmed that metallic Ag nanoparticles were precipitated within glass samples in the laser-written domain. The surface plasmon absorbance fits well with the experimental absorption spectrum. The nonlinear absorption coefficient $\beta$ is determined to be $2.47 \times 10^{-14} \mathrm{~m} / \mathrm{W}$ by fitting the open aperture Z-scan curve, which originated from the intraband transition in the $s-p$ $\mathrm{Ag}$ band. The formation mechanism of Ag-glass nanocomposites is discussed as well.
\end{abstract}

Keywords: nonlinear optical materials; laser materials processing; microstructure fabrication. DOI: 10.3788/COL202119.011901

\section{Introduction}

When interacting with intense incident electromagnetic fields, nonlinear optical materials may produce output fields that differ in frequency, amplitude, or phase from the input field ${ }^{[1]}$. The applications of optical materials with large nonlinear optical coefficients can be found in saturable absorbers ${ }^{[2]}$, optical limiters $^{[3]}$, wavelength converters ${ }^{[4]}$, optical switching ${ }^{[5]}$, secondharmonic imaging ${ }^{[6]}$, electro-optic modulation ${ }^{[7]}$, two-photon imaging $^{[8]}$, etc. The relevant nonlinear optical materials now include organic materials ${ }^{[9]}$, chalcogenide glasses ${ }^{[10]}$, twodimensional inorganic semiconductor materials ${ }^{[11]}$, quantumdots-doped $^{[12]}$ or noble metal-nanoparticle-doped composite glass $^{[13]}$, etc.

Inorganic materials usually have better thermal stability and anti-aging ability compared to organic materials, which have been widely used in industry, military, aerospace, automotive, and civilian facilities. Among inorganic materials, glasses are more easily synthesized and mechanically machined than crystals. Also, composite glass has greater optical nonlinearities than pure glasses, for example, metal-nanoparticles-doped glasses have a quicker response, and $\chi^{(3)}$ is a million times that of fused silica ${ }^{[14]}$ due to the higher free-electron density ${ }^{[15]}$. Metal nanoparticles can enhance the optical nonlinearity of glasses by quantum confinement and local electric field effect near the plasmon resonance frequency. Overall, the third-order nonlinear optical materials requirements include low linear and nonlinear absorption, simple synthesis, thermal stability, convenient mechanical treatment, rapid response, and high nonlinear susceptibility ${ }^{[16]}$. Metal-nanoparticles-doped glasses are promising for fulfilling all of these requirements.

Nonlinear inorganic glasses doped with metal nanoparticles can be prepared conventionally by ion implantation, heat treatment, magnetron sputtering, and sol-gel methods ${ }^{[17-20]}$. Laser treatment has recently attracted a lot of attention since laser provides three-dimensional (3D) spatially selective precipitation of metal nanoparticles in bulk glasses. Previous work focused mainly on fabricating metal nanoparticles in the glass by femtosecond lasers. Successive annealing is typically expected after low-repetition-rate femtosecond laser writing ${ }^{[21,22]}$, which is time-consuming. Metal Ag nanoparticles were directly introduced without successive heat treatment by using a high-repetition-rate femtosecond laser ${ }^{[23-25]}$. However, femtosecond laser writing resulted in micro-voids in the glass matrix ${ }^{[23]}$. Therefore, the transmittance of the glasses was reduced due to the interface reflection in these defects.

In this Letter, we use a high-repetition-rate picosecond laser to directly precipitate Ag nanoparticles in silicate glass, which could reduce the reflection loss caused by structural defects. Also, the solubility of $\mathrm{Ag}^{+}$in silicate glass is usually limited because the maximum concentration in previous studies ${ }^{[22-25]}$ is only $0.1 \%$ (mole fraction). By adding $\mathrm{Sb}_{2} \mathrm{O}_{3}$ in the prepared 
glass samples, the $\mathrm{Ag}^{+}$concentration will increase to six times greater than that of previous studies ${ }^{[22-25]}$.

\section{Experiment}

$\mathrm{Ag}^{+}$-doped silicate glasses were prepared by the conventional melt-quenching technique. The composition of starting raw materials is $4 \mathrm{NaNO}_{3}-16 \mathrm{Na}_{2} \mathrm{CO}_{3}-12.5 \mathrm{CaCO}_{3}-69.5 \mathrm{SiO}_{2}-$ $0.25 \mathrm{NH}_{4} \mathrm{Cl}-1 \mathrm{Sb}_{2} \mathrm{O}_{3}-x \mathrm{AgNO}_{3}$, where $x$ stands for the nominal amount of added $\mathrm{AgNO}_{3}$ in mole fraction. All of the reagents are analytical grade. In the glass system, $\mathrm{NaNO}_{3}, \mathrm{NH}_{4} \mathrm{Cl}$, and $\mathrm{Sb}_{2} \mathrm{O}_{3}$ acted as refining agents. The reagents were thoroughly mixed and filled into crucibles. Each batch was melted for $3 \mathrm{~h}$ under an ambient atmosphere in a resistively heated furnace at $1600^{\circ} \mathrm{C}$. The melts were poured onto a preheated copper plate (at $400^{\circ} \mathrm{C}$ ) and splash-cooled by pressing with another copper plate. The solidified samples were subsequently annealed at $570^{\circ} \mathrm{C}$ for $4 \mathrm{~h}$. Thus, we obtained glass samples with a composition of $18 \mathrm{Na}_{2} \mathrm{O}-12.5 \mathrm{CaO}-69.5 \mathrm{SiO}_{2}-1 \mathrm{Sb}_{2} \mathrm{O}_{3}-x \mathrm{Ag}$ (mole fraction). Depending on the concentration of $\mathrm{AgNO}_{3}$, glass samples are labeled as $\mathrm{S} 1$ to S6, as shown in Table 1.

For the precipitation of $\mathrm{Ag}$ nanoparticles inside silicate glasses, a high-repetition-rate picosecond laser (HE1060, Fianium) was used. The laser was operated at a pulse duration of $100 \mathrm{ps}$, a wavelength of $532 \mathrm{~nm}$, and a repetition rate of $500 \mathrm{kHz}$. A microscope (Nikon Eclipse ME600) with a 50 times objective lens at a numerical aperture of 0.8 was used to focus the laser beam into the bulk of the sample. Glass samples were translated at a precision of $1 \mu \mathrm{m}$ using an XYZ stage (Prior Stage II). A CCD camera (Mshot MC50) equipped on a microscope was used for real-time monitoring.

A UV-visible (VIS)-near-IR (NIR) spectrophotometer (Jasco V570) with a resolution of $0.3 \mathrm{~nm}$ was used to record the absorbance of glass samples in the wavelength range of $200-800 \mathrm{~nm}$. Transmission electron microscopy (TEM) measurement was done by a field emission transmission electron microscope (Tecnai G2 F20 S-TWIN, FEI). The glass transition temperature was measured by a differential scanning calorimeter (Netzsch Sta 409). The Z-scan technique was introduced to measure the optical nonlinearity of glass samples. The femtosecond laser source in the Z-scan measurement was a Ti-sapphire laser (Spectra-Physics, Spitfire), with a repetition rate of $1 \mathrm{kHz}$, a wavelength of $800 \mathrm{~nm}$, and the pulse width is $185 \mathrm{fs}$. A silicon detector (Coherent OP-2 VIS) and a thermal detector (Coherent PM3) separately record the incident and transmitted laser power.

Table 1. Concentrations of Doped- $\mathrm{Ag}^{+}$in the Silicate Glasses.

\begin{tabular}{ccccccc}
\hline Sample & S1 & S2 & S3 & S4 & S5 & S6 \\
\hline Concentration of Ag (\%) & 0 & 0.01 & 0.04 & 0.16 & 0.64 & 2.56 \\
\hline
\end{tabular}

\section{Results and Discussion}

In Fig. 1, we show the fabricated $\mathrm{Ag}^{+}$-doped silicate glass samples and the resulting samples subjected to laser treatment. Samples S1-S5 are colorless, while S6 with higher $x$ value possesses a dull brown coloration, implying Ag precipitation ${ }^{[22]}$. Samples S1-S5 are all irradiated by the picosecond laser with the same parameters with a rectangular region (average power $1.1 \mathrm{~W}$, speed $200 \mu \mathrm{m} / \mathrm{s}$ ). It can be found that the laserirradiated region in S1 remains colorless, while the laser-treated zone in S2-S5 turns yellow, indicating the precipitation of Ag nanoparticles ${ }^{[22]}$. It is quite obvious that the laser-induced color of yellow becomes darker, but is still transparent with increasing $\mathrm{Ag}^{+}$concentrations, which indicates a higher concentration of Ag nanoparticles.

Therefore, we can infer that by adding $\mathrm{Sb}_{2} \mathrm{O}_{3}$, at least $0.64 \%$ of silver ions will dissolve in this form of silicate glass, and this dissolving concentration is six times greater than that of previous studies $(0.1 \%)^{[22-25]}$. However, there is not any theoretical explanation that we can refer to so far. We assume that $\mathrm{Ag}$ can hardly break the $\mathrm{Si}-\mathrm{O}-\mathrm{Si}$ bond, thus restricting the dissolving $\mathrm{Ag}^{+}$concentration. However, the glass network former $\mathrm{Sb}_{2} \mathrm{O}_{3}$ possesses a triangle structure, so the $\mathrm{Sb}-\mathrm{O}-\mathrm{Sb}$ bond can be broken more easily by Ag atoms, as shown in Fig. $2^{[26]}$. In this way, more $\mathrm{Ag}^{+}$could be dissolved in the glass matrix.

Absorption spectra before and after irradiation by picosecond laser pulses are illustrated in Fig. 3. For precursor glass, the absorption spectra of S1-S4 are almost overlapped due to the low concentration of Ag [Fig. 3(a)]. After laser irradiation, the absorption intensity of all of the samples is significantly enhanced, as shown in Fig. 3(b).

An obvious absorbance peak of sample S5 appears, whereas no absorbance peak is ever found for S1, S2, S3, and S4. From the difference spectra of the glass samples before and after laser irradiation, we can see a more distinct absorption peak around $430 \mathrm{~nm}$, which is a typical absorption band of $\mathrm{Ag}$ nanoparticles ${ }^{[23]}$.

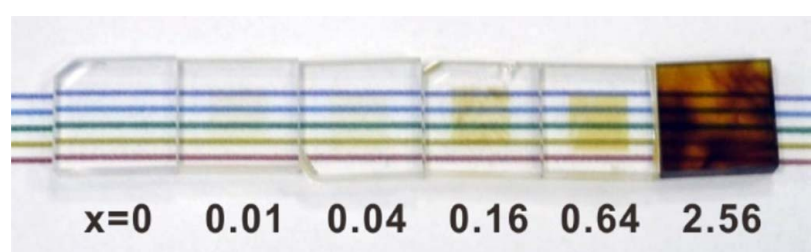

Fig. 1. Photographs of $\mathrm{Ag}^{+}$-doped silicate glasses with a rectangular region processed by a picosecond laser at an average power of $1.1 \mathrm{~W}$ and a scanning speed of $200 \mu \mathrm{m} / \mathrm{s}$.

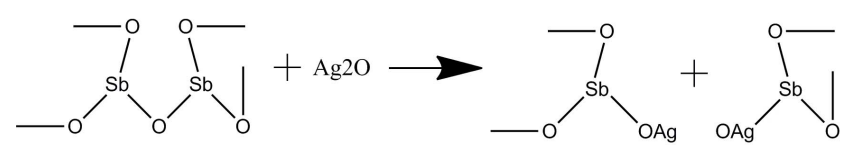

Fig. 2. Ag atoms dissolve in the glass network by breaking the Sb-0-Sb bond. 


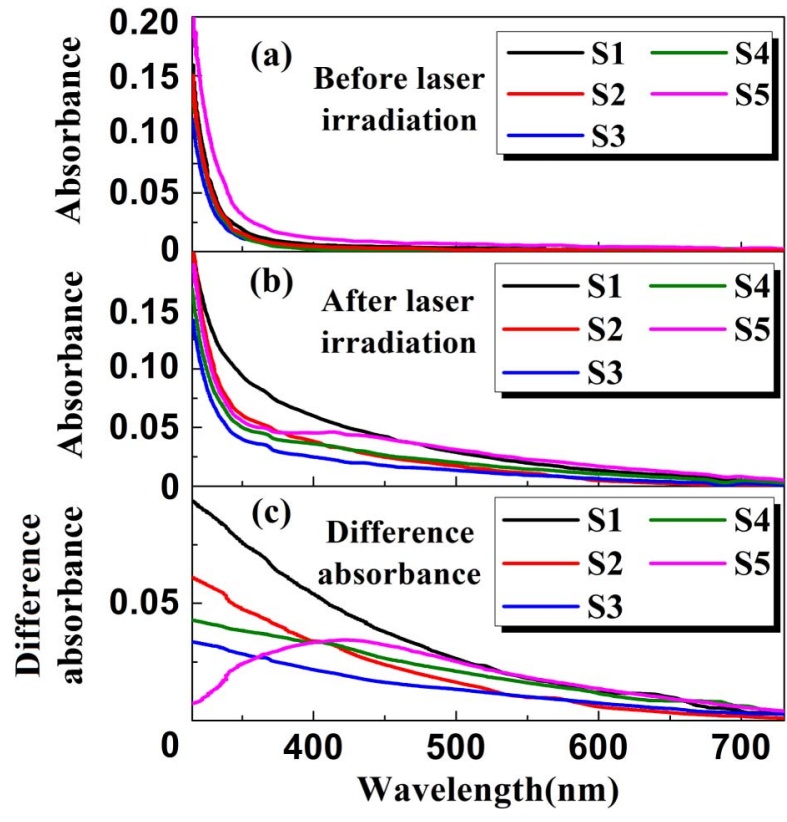

Fig. 3. Absorption spectra of five glass samples (a) before and (b) after picosecond laser irradiation; (c) difference absorbance after picosecond laser irradiation.

Theoretical absorbance of silicate glass with Ag nanoparticles precipitated in it is also determined in addition to the measured absorbance of precipitated Ag nanoparticles. Based on the Maxwell-Garnett theory, the absorbance coefficient $\alpha$ is calculated using Eq. (1):

$$
\alpha(\omega)=\frac{2 \omega}{c} \operatorname{Im} \sqrt{\epsilon_{\text {eff }}(\omega)},
$$

where the effective dielectric constant $\varepsilon_{\text {eff }}(\omega)$ of Ag nanoparticles contained in silicate glass is expressed as $\varepsilon_{\text {eff }}(\omega)=$ $\varepsilon_{h} \frac{\left(\varepsilon_{i}+2 \varepsilon_{h}\right)+2 \eta\left(\varepsilon_{i}-\varepsilon_{h}\right)}{\left(\varepsilon_{i}+2 \varepsilon_{h}\right)-2 \eta\left(\varepsilon_{i}-\varepsilon_{h}\right)}$ and $\varepsilon_{i}(\omega)=\varepsilon_{b}+1-\frac{\omega_{p}^{2}}{\omega^{2}+i \gamma \omega}$, where plasmon frequency $\omega_{p}$ is $1.4 \times 10^{16} \mathrm{~Hz}$, free-electron damping constant $\gamma$ is $1 \times 10^{15} \mathrm{~Hz}$, and dielectric constants of metal $\varepsilon_{b}$ and surrounding glass matrix $\varepsilon_{h}$ are 4.2 and 2.34 , respectively ${ }^{[27]}$. The volume ratio $\eta$ of $\mathrm{Ag}$ nanoparticles could be calculated by $\eta=V_{\mathrm{Ag}} / \sum V_{i}=\left(m_{\mathrm{Ag}} / \rho_{\mathrm{Ag}}\right) /\left(\sum m_{i} / \rho_{\text {glass }}\right)$, where $m_{\mathrm{Ag}}$, $V_{\mathrm{Ag}}$, and $\rho_{\mathrm{Ag}}$ represent the mass, volume, and density of Ag, while $m_{i}, V_{i}$, and $\rho_{\text {glass }}$ are the mass, volume, and density of the final glass sample. As the dissolving concentration of $\mathrm{Ag}$ in the glass sample is quite low, we assume that the volume ratio of $\mathrm{Ag}$ nanoparticles is equal to the volume ratio of $\mathrm{Ag}$ ions. Therefore, $\eta$ in S5 is about $0.27 \%$.

In this model, absorbance is proportional to $\alpha$ with an unknown factor. By selecting an appropriate multiplier, theoretical absorbance is fitted in Fig. 4(a). Comparing the theoreti$\mathrm{cal}$ and measured absorbance in Fig. 4(a), the position and shape of the surface plasmon resonance (SPR) can be seen to fit well with the experimental absorption of silicate glass with Ag nanoparticles. The experimental absorbance peak is around $416 \mathrm{~nm}$,

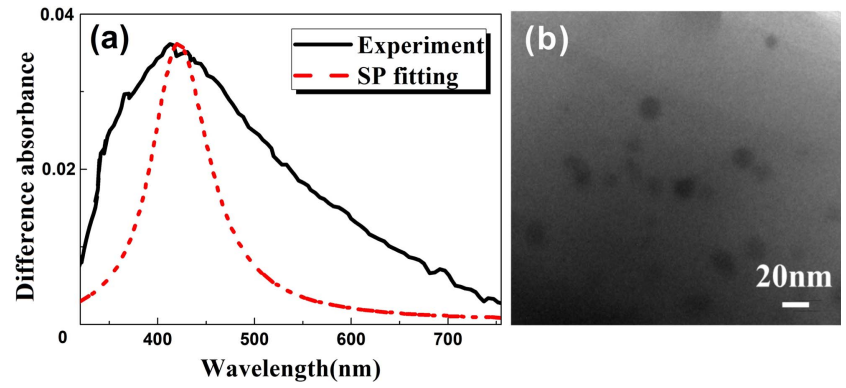

Fig. 4. (a) Experimental difference spectrum and theoretical fitting curve of Ag nanoparticle SPR of S5; (b) TEM image of Ag nanoparticles precipitated in the laser-irradiated zone of S5.

and the resonant wavelength of $\mathrm{Ag}$ nanoparticles is at $424 \mathrm{~nm}$. However, the full width at half-maximum of the experimental absorption is wider than the calculated value. This difference can be attributed to the size dispersion of $\mathrm{Ag}$ nanoparticles and the change of absorption of the microstructures.

TEM observes Ag nanoparticles in silicate glasses. Ag aggregates into crystal nuclei and grows into nanoparticles due to the heat accumulation of picosecond laser pulses. As seen in Fig. 4(b), the diameter of $\mathrm{Ag}$ nanoparticle ranges from $9 \mathrm{~nm}$ to $19 \mathrm{~nm}$, and the average diameter is about $14 \mathrm{~nm}$.

The peak power density of the picosecond laser is a key factor for the precipitation of Ag nanoparticles in silicate glass. The picosecond laser could not induce microstructures until the average power is increased to $0.86 \mathrm{~W}$. The diffraction-limited spot size of the laser $\left(2 \omega_{0}\right)$ can be estimated as $2 \omega_{0}=$ $4 \lambda M^{2} f / \pi D$, where $M^{2}$ is the beam quality factor, which is about 1.5 in this experiment, $\lambda$ is the wavelength of the laser beam, $f$ is the focal length of the objective lens with the value of $4 \mathrm{~mm}, D$ is the beam diameter, and its value is $2.5 \mathrm{~mm}$. Therefore, the laser beam is focused on a spot size of about $2 \omega_{0}=1.62 \mu \mathrm{m}$ in diameter. Thus, the critical peak power density for precipitating $\mathrm{Ag}$ nanoparticles is about $3.3 \times 10^{12} \mathrm{~W} / \mathrm{cm}^{2}$.

In addition to peak power density, laser scanning speed also plays an important role in the precipitation of $\mathrm{Ag}$ nanoparticles. Figure 5 shows the optical micrographs of the laser-induced seven lines in S5 with the laser scanning speed range of 16, $32,64, \ldots, 1024 \mu \mathrm{m} / \mathrm{s}$ at an average power of $1.1 \mathrm{~W}$. Micro-voids are observed when scanning speed is 16 and $32 \mu \mathrm{m} / \mathrm{s}$, which is caused by the high pressure induced by multiple picosecond laser pulses. When the scanning speed ranges from 64 to $512 \mu \mathrm{m} / \mathrm{s}$, Ag nanoparticles are precipitated homogeneously in the glass matrix. However, with the laser moving faster than $1024 \mu \mathrm{m} / \mathrm{s}$, laser-induced heat is not sufficiently high, so only a small portion of $\mathrm{Ag}$ aggregates into nanoparticles. Thus, the color is relatively light. So, for TEM and absorption measurement, a scanning speed of $200 \mu \mathrm{m} / \mathrm{s}$ was chosen. The inset photograph of Fig. 5 shows an obvious color contrast of the laser fabricated region in S5 at speeds of 200 and $1000 \mu \mathrm{m} / \mathrm{s}$.

A previous study shows that in top-view microscope images of the femtosecond laser-irradiated region, it appears as a ring. Ag aggregated in the center of the ring-shaped region, namely in 


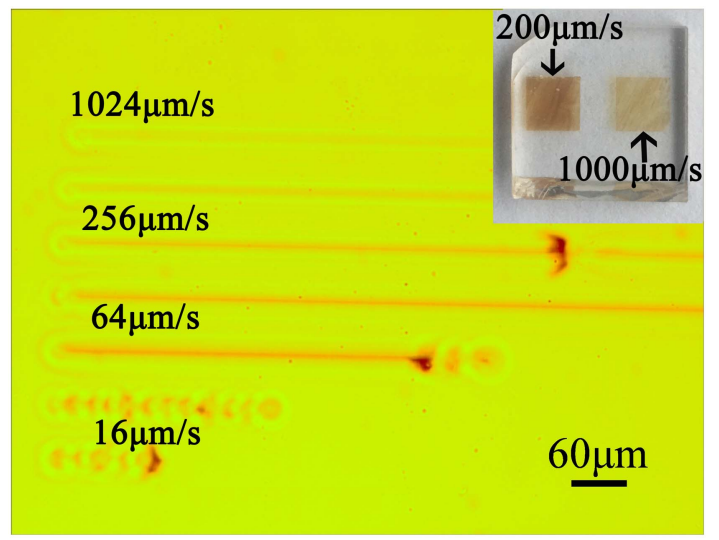

Fig. 5. Optical micrographs of scanned lines by picosecond laser pulses at different processing speeds of $16,32,64, \ldots, 1024 \mu \mathrm{m} / \mathrm{s}$. The inset shows the color comparison of the laser fabricated region in S5 at speeds of 200 and $1000 \mu \mathrm{m} / \mathrm{s}$.

the micro-void. The femtosecond laser transforms the material in the focal point into plasma and generates a fast-outward propagating shockwave, which leaves micro-voids in silicate glass. The micro-void was mainly caused by the electrical repulsive force between a mass of electrons, and the micro-void interface reflection will reduce the transmittance of glass. The different absorption peaks of the glass sample before and after femtosecond laser treatment are therefore as high as about $0.22^{[23]}$. However, the picosecond laser has a longer pulse duration, thus pulse energy can be transferred to a lattice ${ }^{[28]}$ and absorbed to melt glass before plasma comes into being, which avoids the formation of micro-voids. So, we observed a much lower absorption peak of about 0.036 in Fig. 4(a). Compared with a femtosecond laser, picosecond laser fabrication can greatly decrease the linear absorption of $\mathrm{Ag}^{+}$-doped glass. $\mathrm{Ag}$ nanoparticles precipitation in silicate glass by picosecond lasers is, therefore, more suitable for real applications.

After Ag nanoparticles were precipitated in S5, its nonlinear optical property was measured using the Z-scan experimental technique. The normalized transmittance is described in Eq. $(2)^{[29]}$ :

$$
T(z)=\sum_{m=0}^{\infty} \frac{\left[-q_{0}(z)\right]^{m}}{(1+m)^{3 / 2}} \approx 1-2^{-3 / 2} q_{0}(z),
$$

where $\quad q_{0}(z)=\beta I_{0} L_{\text {eff }} /\left(1+z^{2} / z_{0}^{2}\right), \quad L_{\text {eff }}=[1-\exp (-\alpha L)] / \alpha$, and $I_{0}=P /\left(R \tau \pi \omega_{1}^{2}\right)$. Rayleigh distance $z_{0}$ is $2.95 \mathrm{~mm}$. Sample thickness $L$ is $0.8 \mathrm{~mm}$. The linear absorption coefficient $\alpha$ of irradiated glass $\mathrm{S} 5$ is $516 \mathrm{~m}^{-1}$. From the experimental waist radius, $\omega_{1}$ is $31.4 \mu \mathrm{m}$, and the pulse duration of femtosecond laser $\tau$ is 131 fs. $P$ and $R$ represent the incident power and repetition rate of a femtosecond laser, respectively.

According to Eq. (2), at the laser focus $(z=0)$, the normalized transmittance has a linear relation with peak intensity, and the slope is $2^{-3 / 2} \beta L_{\text {eff }}$, as shown in Eq. (3):
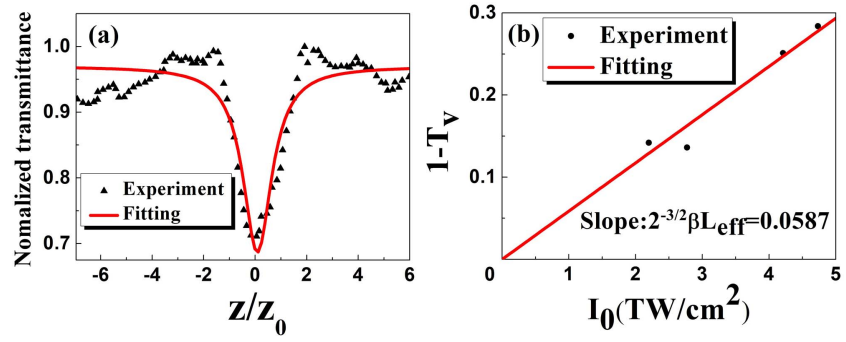

Fig. 6. (a) Normalized Z-scan transmittance of $\mathrm{S} 5$ at $I_{0}=4.22 \mathrm{TW} / \mathrm{cm}^{2}$; (b) the dependence of Z-scan valley transmittance on incident laser intensity $l_{0}$.

$$
1-T_{v}=1-T(z=0)=2^{-3 / 2} \beta L_{\mathrm{eff}} I_{0}
$$

where $T_{v}$ is the valley transmittance of the Z-scan plot.

Figure 6 (a) shows the normalized Z-scan transmittance of S5 at $I_{0}=4.22 \mathrm{TW} / \mathrm{cm}^{2}$, and the Z-scan valley transmittance at different $I_{0}$ is also recorded in Fig. 6(b). According to Eq. (3), the nonlinear absorption coefficient $\beta$ of irradiated glass $\mathrm{S} 5$ is calculated to be $2.47 \times 10^{-14} \mathrm{~m} / \mathrm{W}$. The nonlinear optical absorption is proposed to the intraband transition in the $s-p$ band of $\mathrm{Ag}$ instead of hot electrons and interband transition ${ }^{[30]}$. The hot electron effect can be neglected because the wavelength of the femtosecond laser is far from SPR. Interband transition does not happen, as the energy of two photons of the femtosecond laser is smaller than the narrowest interband gap $(3.45 \mathrm{eV})$ of $\mathrm{Ag}^{[31]}$.

\section{Conclusion}

Space selective precipitation of Ag nanoparticles in silicate glass was achieved by $500 \mathrm{kHz}$ picosecond laser pulses without further heat treatment. By adding $\mathrm{Sb}_{2} \mathrm{O}_{3}, \mathrm{Ag}$ concentration has been improved by six times in silicate glass. Linear absorbance was reduced to 0.0364 by preventing the formation of micro-voids. Nonlinear absorption coefficient $\beta$ is determined to be $2.47 \times$ $10^{-14} \mathrm{~m} / \mathrm{W}$ by fitting the open aperture Z-scan curve, which originates from an intraband transition in the $s-\mathrm{p}$ band of $\mathrm{Ag}$.

\section{Acknowledgement}

This work was supported by the National Key Research and Development Program of China (No. 2016YFB1102405), National Natural Science Foundation of China (No. 61675214), and Shanghai Sailing Program (No. 20YF1455200).

\section{References}

1. D. Eaton, "Nonlinear optical materials," Science 253, 281 (1991).

2. L. Yun, Y. Qiu, C. Yang, J. Xing, K. Yu, X. Xu, and W. Wei, "PbS quantum dots as a saturable absorber for ultrafast laser," Photon. Res. 6, 1028 (2018).

3. G. Zhou and W. Wong, "Organometallic acetylides of $\mathrm{Pt}^{\mathrm{II}}, \mathrm{Au}^{\mathrm{I}}$ and $\mathrm{Hg}^{\mathrm{II}}$ as new generation optical power limiting materials," Chem. Soc. Rev. 40, 2541 (2011). 
4. K. Inoue and H. Toba, "Wavelength conversion experiment using fiber fourwave mixing," IEEE Photon. Technol. Lett. 4, 69 (1992).

5. M. Y. Shubar, H. L. Saadon, and S. J. Abbas, "Nonlinear optical switching and all-figures of merit in $\mathrm{Bi}_{2} \mathrm{~S}_{3-x} \mathrm{Se}_{x} / \mathrm{PMMA}$ nanocomposite films investigated by Z scan under visible CW laser," Chin. Opt. Lett. 18, 011902 (2020).

6. G. Bautista, J. Makitalo, Y. Chen, V. Dhaka, M. Grasso, L. Karvonen, H. Jiang, M. J. Huttunen, T. Huhtio, and H. Lipsanen, "Second-harmonic generation imaging of semiconductor nanowires with focused vector beams," Nano Lett. 15, 1564 (2015).

7. W. Heni, Y. Kutuvantavida, C. Haffner, H. Zwickel, C. Kieninger, S. Wolf, M. Lauermann, Y. Fedoryshyn, A. F. Tillack, and L. E. Johnson, "Siliconorganic and plasmonic-organic hybrid photonics," ACS Photon. 4, 1576 (2017).

8. Y. I. Park, K. T. Lee, Y. D. Suh, and T. Hyeon, "Upconverting nanoparticles: a versatile platform for wide-field two-photon microscopy and multi-modal in vivo imaging," Chem. Soc. Rev. 44, 1302 (2015).

9. J. L. Bredas, C. Adant, P. Tackx, A. Persoons, and B. Pierce, "Third-order nonlinear optical response in organic materials: theoretical and experimental aspects," Chem. Rev. 94, 243 (1994).

10. X. Wang, Z. Wang, J. Yu, C. Liu, X. Zhao, and Q. Gong, "Large and ultrafast third-order optical nonlinearity of $\mathrm{GeS}_{2}-\mathrm{Ga}_{2} \mathrm{~S}_{3}$-CdS chalcogenide glass," Chem. Phys. Lett. 399, 230 (2004).

11. M. He, Y. Chen, L. Zhu, H. Wang, X. Wang, X. Xu, and Z. Ren, "Third-order nonlinear optical properties of $\mathrm{WTe}_{2}$ films synthesized by pulsed laser deposition," Photon. Res. 7, 1493 (2019).

12. Z. Zhang, Z. Lü, X. Yang, H. Chai, L. Meng, and T. Yang, " $25 \mathrm{~Gb} / \mathrm{s}$ directly modulated ground-state operation of $1.3 \mu \mathrm{m}$ InAs/GaAs quantum dot lasers up to $75^{\circ} \mathrm{C}$," Chin. Opt. Lett. 18, 071401 (2020).

13. M. Brust, D. Bethell, and C. J. Kiely, "Self-assembled gold nanoparticle thin films with nonmetallic optical and electronic properties," Langmuir 14, 5425 (1998).

14. K. Uchida, S. Kaneko, S. Omi, C. Hata, H. Tanji, Y. Asahara, A. J. Ikushima, T. Tokizaki, and A. Nakamura, "Optical nonlinearities of a high concentration of small metal particles dispersed in glass: copper and Ag particles," J. Opt. Soc. Am. B 11, 1236 (1994).

15. M. Yamane and Y. Asahara, Glasses Photonics (Cambridge University, 2005).

16. A. J. Almosawe and H. L. Saadon, "Nonlinear optical and optical limiting properties of new structures of organic nonlinear optical materials for photonic applications," Chin. Opt. Lett. 11, 041902 (2013).

17. B. Can-Uc, R. Rangel-Rojo, L. Rodriguez-Fernandez, and A. Oliver, "Polarization selectable nonlinearities in elongated $\mathrm{Ag}$ nanoparticles embedded in silica," Opt. Mater. Express. 3, 2012 (2013).
18. G. Lin, D. Tan, F. Luo, D. Chen, Q. Zhao, and J. Qiu, "Linear and nonlinear optical properties of glasses doped with Bi nanoparticles," J. Non. Cryst. Solids 357, 2312 (2011).

19. E. Danielson, V. Dhamodharan, and A. Porkovich, "Gas-phase synthesis for label-free biosensors: zinc-oxide nanowires functionalized with gold nanoparticles," Sci. Rep. 9, 17370 (2019).

20. P. P. Kiran, B. N. S. Bhaktha, D. N. Rao, and G. De, "Nonlinear optical properties and surface-plasmon enhanced optical limiting in $\mathrm{Ag}-\mathrm{Cu}$ nanoclusters co-doped in $\mathrm{SiO}_{2}$ Sol-Gel films," J. Appl. Phys. 96, 6717 (2004).

21. J. Qiu, X. Jiang, C. Zhu, M. Shirai, J. Si, N. Jiang, and K. Hirao, "Manipulation of gold nanoparticles inside transparent materials," Angew. Chem. Int. Ed. Engl. 43, 2230 (2004).

22. J. Qiu, M. Shirai, T. Nakaya, J. Si, X. Jiang, C. Zhu, and K. Hirao, "Spaceselective precipitation of metal nanoparticles inside glasses," Appl. Phys. Lett. 81, 3040 (2002).

23. N. Ma, H. Ma, M. Zhong, J. Yang, Y. Dai, G. Ye, Z. Yue, G. Ma, and J. Qiu, "Direct precipitation of Ag nanoparticles induced by a high repetition femtosecond laser," Mater. Lett. 63, 151 (2009).

24. J. Guo, B. Hua, G. Qian, M. Wang, J. Si, J. Qiu, and K. Hirao, "Direct space selective precipitation of Ag nanoparticles inside silicate glasses through local heating of erbium," J. Alloys Compd. 468, 563 (2009).

25. Y. Dai, G. Yu, M. He, H. Ma, X. Yan, and G. Ma, "High repetition rate femtosecond laser irradiation-induced elements redistribution in Ag-doped glass," Appl. Phys. B 103, 663 (2011).

26. J. Ashok, M. Kostrzewa, A. Ingram, N. Venkatramaiah, M. S. Reddy, V. R. Kumar, M. Piasecki, and N. Veeriaiah, "Structural and dielectric features of silver doped sodium antimonate glass ceramics," J. Alloys Compd. 791, 278 (2019).

27. A. Podlipensky, A. Abdolvand, G. Seifert, and H. Graener, "Femtosecond laser assisted production of dichroitic 3D structures in composite glass containing Ag nanoparticles," Appl. Phys. A 80, 1647 (2004).

28. B. Chichkov and C. Momma, "Femtosecond, picosecond and nanosecond laser ablation of solids," Appl. Phys. A 63, 109 (1996).

29. M. Sheik-Bahae, A. A. Said, J. D. Hagen, and E. W. Van Stryland, "Sensitive measurement of optical nonlinearities using a single beam," IEEE J. 26, 760 (1990).

30. M. Yamane and Y. Asahara, Glasses for Photonics (Cambridge University, 2000).

31. N. Christensen, "The band structure of Ag and optical interband transitions," Phys. Status Solid. 54, 551 (1972). 